\section{IN THE NEWS}

\section{Mental metal}

Iron supplements can improve the cognitive performance of even mildly iron-deficient women, according to data presented at the Experimental Biology meeting in Washington DC.

John Beard, Laura MurrayKolb and colleagues from

Pennsylvania State University tested the attention, memory and learning capacity of women aged 18-35.

According to Beard, "The iron-deficient women took longer to do tasks and were less successful." The performance of anaemic women was even worse. "The number of errors in a memory task was double than in the iron-sufficient group" (New Scientist, 20 April).

"The good news is that replacement does help functioning return to normal," Murray-Kolb told WebMD

(19 April). The performance of iron-deficient women who took 60 milligrams of iron every day improved after four months. "It was a highly significant improvement, which is really the proof of the pudding," said Beard (New Scientist).

Iron deficiency is linked to altered brain chemistry in rats, and the team from Penn State suggest that a similar effect could underlie the impaired cognitive ability of women that lack this essential mineral. Beard told New Scientist that "Iron deficiency could have an adverse impact on the brain by affecting the dopamine and serotonin neurotransmitter systems."

Boosting iron levels might normalize production of these chemical messengers, thereby maximizing the efficiency of learning and memory.

"Women need to know this actually is affecting their brain and the way they're thinking," said Murray-Kolb

(HealthDayNews, 19 April). But she cautions against rushing out to the pharmacy. Women with normal iron levels will not benefit from supplements, and excess iron can be harmful to the heart and liver.

Suzanne Farley
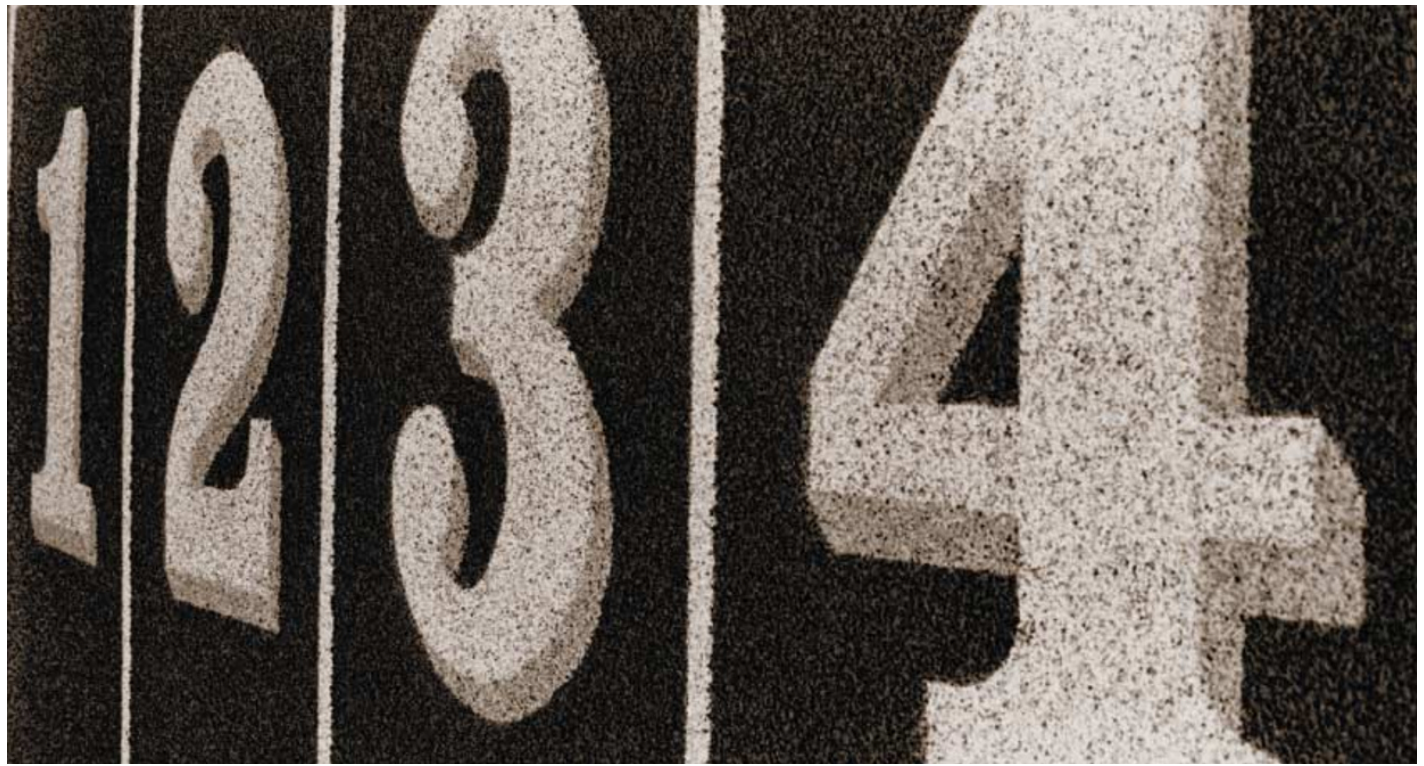

ION CHANNELS

\title{
Counting CaMs at calcium channels
}

How many calmodulin (CaM) molecules does it take to turn off a calcium channel? It sounds like the start to a bad joke, but Mori and colleagues have tackled this question using channels fused to CaM molecules and the answer, they say, is just one.

CaM interacts with calcium channels to carry out two functions: it mediates calcium-dependent inactivation of the channels, and it transduces the signal from calcium entry through the channels into various intracellular signalling pathways. Mori et al. fused CaM molecules to L-type calcium channels to investigate the stoichiometry of these processes.

In the absence of calcium, CaM molecules preassociate with L-type calcium channels. When calcium subsequently enters through the channel and interacts with $\mathrm{CaM}$, the channel is inactivated. The authors used polyglycine linkers to fuse an inactive, mutant $\mathrm{CaM}$ $\left(\mathrm{CaM}_{\mathrm{MUT}}\right)$ to each L-type calcium channel, on the principle that the $\mathrm{CaM}_{\mathrm{MUT}}$ would act as a dominantnegative and prevent wild-type $\mathrm{CaM}$ from associating with the channel. Channel-CaM ${ }_{\mathrm{MUT}}$ chimaeras did not undergo calcium-dependent inactivation, but channels fused to a wild-type CaM did, indicating that the fused $\mathrm{CaM}$ is important for inactivation.

To test whether other CaM molecules were needed for inactivation to occur, Mori et al. overexpressed free $\mathrm{CaM}_{\text {MUT }}$ along with L-type calcium channels. Unfused channels showed no inactivation in these circumstances, because the $\mathrm{CaM}_{\mathrm{MUT}}$ was occluding the pre-association site and preventing wild-type $\mathrm{CaM}$ from inactivating the channels. However, channels that were fused to a wild-type CaM showed normal inactivation, demonstrating that a single $\mathrm{CaM}$ molecule is both necessary and sufficient for calciumdependent inactivation.
In another part of the study, the authors investigated the local concentration of endogenous $\mathrm{CaM}$ around channels. They took advantage of the variable lengths of polyglycine linkers. When short linkers are used, $\mathrm{CaM}_{\mathrm{MUT}}$ completely occludes the pre-association sites and calcium-dependent inactivation is blocked. With longer linkers, however, the local concentration of $\mathrm{CaM}_{\mathrm{MUT}}$ drops, allowing endogenous $\mathrm{CaM}$ to access the preassociation sites and to mediate calcium-dependent inactivation. This effect can be used to estimate the local concentration of endogenous CaM near the channels. The authors calculated that the level of local endogenous $\mathrm{CaM}$ at the pre-association locus was around $2.5 \mathrm{mM}-$ much higher than in the cell as a whole.

Such local enrichment of CaM near L-type calcium channels should allow the calcium influx through one channel to activate many CaM molecules, so that either many signalling pathways can be activated or a single pathway can be amplified.

These insights into the stoichiometry of channel regulation and signalling by $\mathrm{CaM}$ should help to advance our understanding of the mechanisms by which CaM carries out these functions. The approach of linking molecules to investigate their interactions should also be a valuable technique for researchers in other fields, and will no doubt produce many more fascinating results.

Rachel Jones

\section{(1) References and links}

ORIGINAL RESEARCH PAPER Mori. M. X. et al. Functional stoichiometry and local enrichment of calmodulin interacting with $\mathrm{Ca}^{2+}$ channels. Science 304, 432-435 (2004)

FURTHER READING Budde, $T$. et al. Calcium-dependent inactivation of neuronal calcium channels. Nature Rev. Neurosci. 3, 873-883 (2002) 